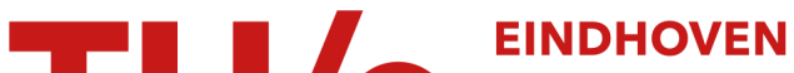 \\ UNIVERSITY OF \\ TECHNOLOGY
}

\section{Short time behavior in de gennes' reptation model}

\section{Citation for published version (APA):}

Ebert, U., Baumgärtner, A., \& Schäfer, L. (1997). Short time behavior in de gennes' reptation model. Physical Review Letters, 78(8), 1592-1595. https://doi.org/10.1103/PhysRevLett.78.1592

DOI:

10.1103/PhysRevLett.78.1592

Document status and date:

Published: 01/01/1997

\section{Document Version:}

Publisher's PDF, also known as Version of Record (includes final page, issue and volume numbers)

\section{Please check the document version of this publication:}

- A submitted manuscript is the version of the article upon submission and before peer-review. There can be important differences between the submitted version and the official published version of record. People interested in the research are advised to contact the author for the final version of the publication, or visit the $\mathrm{DOI}$ to the publisher's website.

- The final author version and the galley proof are versions of the publication after peer review.

- The final published version features the final layout of the paper including the volume, issue and page numbers.

Link to publication

\section{General rights}

Copyright and moral rights for the publications made accessible in the public portal are retained by the authors and/or other copyright owners and it is a condition of accessing publications that users recognise and abide by the legal requirements associated with these rights.

- Users may download and print one copy of any publication from the public portal for the purpose of private study or research.

- You may not further distribute the material or use it for any profit-making activity or commercial gain

- You may freely distribute the URL identifying the publication in the public portal.

If the publication is distributed under the terms of Article 25fa of the Dutch Copyright Act, indicated by the "Taverne" license above, please follow below link for the End User Agreement:

www.tue.nl/taverne

Take down policy

If you believe that this document breaches copyright please contact us at:

openaccess@tue.nl

providing details and we will investigate your claim. 


\title{
Short Time Behavior in de Gennes' Reptation Model
}

\author{
Ute Ebert, ${ }^{1,3}$ Artur Baumgärtner, ${ }^{2}$ and Lothar Schäfer ${ }^{3}$ \\ ${ }^{1}$ Instituut-Lorentz, Universiteit Leiden, Postbus 9506, 2300 RA Leiden, The Netherlands \\ ${ }^{2}$ Institut für Festkörperforschung and Forum Modellierung, Forschungszentrum Jülich, 52425 Jülich, Germany \\ ${ }^{3}$ Fachbereich Physik, Universität Essen, 45117 Essen, Germany
}

(Received 7 August 1996)

\begin{abstract}
To establish a standard for the distinction of reptation from other modes of polymer diffusion, we analytically and numerically study the displacement of the central bead of a chain diffusing through an ordered obstacle array for times $t<\mathcal{O}\left(N^{2}\right)$. Our theory and simulations agree quantitatively and show that the second moment approaches the $t^{1 / 4}$ power law (often viewed as a signature of reptation) only after a very long transient and only for long chains $(N>100)$. Our analytically solvable model furthermore predicts a very short transient for the fourth moment. This is verified by computer experiment. [S0031-9007(97)02510-6]
\end{abstract}

PACS numbers: 83.20.Fk, 05.40.+j, 83.20.Jp

Since the reptation model was formulated 25 years ago by de Gennes [1], it has become a widely used concept of polymer motion. In its original form it is concerned with the diffusion of a mobile polymer chain through a net of impenetrable and immobile obstacles. It describes this motion as being confined to a tube created by the surrounding obstacles. Diffusion takes place by the motion of little wiggles of surplus length, the "defects", along the tube. Asymptotically this scenario yields simple power laws for various quantities [1]. For instance, the diffusion constant $D$, which governs the motion of the total chain at large time scales, should behave as $D \propto N^{-2}$, where $N$ is the chain length, i.e., the polymerization index of the macromolecule. Another important prediction concerns the motion of the central bead $\mathbf{r}_{N / 2}(t)$. For intermediate times it is predicted to diffuse a mean square distance

$$
\begin{aligned}
g_{1}(t) & =\left\langle\left(\mathbf{r}_{N / 2}(t)-\mathbf{r}_{N / 2}(0)\right)^{2}\right\rangle \\
& \propto \begin{cases}t^{1 / 4} & \text { for } T_{0} \ll t \ll T_{2} \\
t^{1 / 2} & \text { for } T_{2} \ll t \ll T_{3} .\end{cases}
\end{aligned}
$$

Here $T_{0}=\mathcal{O}\left(N^{0}\right)$ is a microscopic time and $T_{2}=\mathcal{O}\left(N^{2}\right)$ is the Rouse time, i.e., the characteristic scale over which an unconstrained chain of length $N$ equilibrates. The "reptation time" $T_{3}=\mathcal{O}\left(N^{3}\right)$ marks the onset of the asymptotic diffusional regime, where the central bead just follows the diffusion of the center of mass: $g_{1}(t)=D t$. For comparison, the Rouse model for a free noninteracting chain yields $g_{1}(t) \propto t^{1 / 2}$ for $t \ll T_{2}$ and asymptotic diffusion of both central bead and center of mass with diffusion constant $D \propto N^{-1}$ for $t \gg T_{2}$.

From the beginning, the reptation model has also been applied to polymer motion through melts, dense solutions, or gels, even though in such systems the surrounding medium more or less can relax. A lot of experimental or computer experimental work has aimed at verifying the reptation predictions in such systems. (See [2] for a recent review.) The outcome of these efforts today is somewhat ambiguous. The expected power laws have never been established beyond doubt. Compared to the free motion of a chain, one typically finds some slowing down, giving rise to effective power laws, with no obvious unique and simple interpretation [3,4]. $g_{1}(t)$, for instance, typically behaves as

$$
g_{1}(t) \propto t^{x} \text { with } 0.25<x \lessgtr 0.4 \text { for } T_{0} \lesssim t \lesssim T_{2} .
$$

It is never clear whether such deviations from predictions like (1) are due to the relaxation of the surrounding medium or are intrinsic to the reptation scenario in the available range of chain lengths. Furthermore, it must be stressed that contrary to statements often found in the literature, also in a frozen disordered environment the asymptotic reptation behavior has not been properly observed. In fact, recent work [5-7] suggests that even strictly immobile obstacles can ruin the reptational power laws, due to their disordered spatial distribution. This finding is in contrast to the view prevailing in the older literature. (See [8], for instance.)

In view of this situation, it is somewhat surprising to note that little effort has been made to clarify the implications of the original reptation model, considering polymer diffusion in an ordered array of fixed obstacles. Clearly only such work can provide the basis for a controlled analysis of effects of disorder or relaxation of the surrounding medium. We are aware of only two such studies. In early work [8] Evans and Edwards claimed to find the behavior (1), but the statistics of their data are insufficient for a convincing analysis. In fact, our extended study of their model as presented below clearly demonstrates that for their chain lengths the $t^{1 / 4}$ power law is not yet attained. More recently Deutsch and Madden [9] reconsidered the model and found $D \propto N^{-2.5}$ for $N \lesssim 100$, in contrast to the expected $N^{-2}$ power law.

In order to establish the predictions of the pure reptation model, which serves as a starting point for the investigation of more complicated environments, we here present results of an extended study of reptation through an ordered array of fixed obstacles in three dimensions. Concentrating on the internal motion in a time regime 
prior to free diffusion, we present new extensive simulations, and we introduce an analytically solvable model that modifies the original reptation model of de Gennes [1] to take the discreteness of the chain into account. We find quantitative agreement among our theory and our data. Simple power laws, however, are found only after a surprisingly long initial transient and are reached only by quite long chains. Our theory explains the long transient quantitatively as a consequence of the finite segment size. Furthermore, our theory suggests to determine another quantity, not measured previously, which should show the reptational power laws more clearly, without the long transient. This prediction is also verified by computer experiment. In summary, we provide an analytically understood and numerically tested standard for reptation of discrete chains of finite length, relevant for the interpretation of all previous simulation results, and merging asymptotically $(N \longrightarrow \infty, t \gg 1)$ with the earlier predicted power laws (1) [1]. In future work, this should allow for a meaningful analysis of the influence of disorder or of some slow relaxation of the medium on reptation.

In our simulations we use the Evans-Edwards model [8]: The chain is confined to a cubic lattice. A second identical lattice is taken to represent the obstacles and is placed such that the lattice points coincide with the centers of the cells of the first lattice. Then the only moves allowed are those of "hairpins", which can rotate among lattice bonds (see Fig. 1). This model incorporates the smallest possible tube diameter. It therefore shows the strongest restriction of chain motion and should exhibit reptation in clearest form. In our numerical experiment we concentrated on the short-time behavior (1), searching for the $t^{1 / 4}$ power law. We note, however, that for shorter chains $(N \leq 100)$ we also covered times $t>T_{3}$ and recovered the results of [9]. A full account of our results will be published elsewhere.

Figure 2 shows $g_{1}(t)$ for times $t \lesssim T_{2}$. Obviously, the expected power law $g_{1}(t) \propto t^{1 / 4}$ indeed is attained,

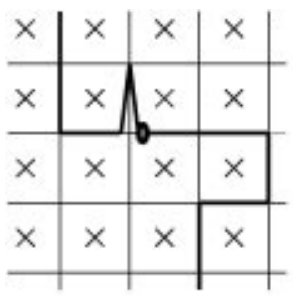

a)

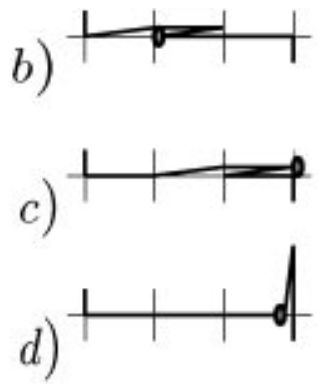

FIG. 1. The Evans-Edwards model: (a) Section of a random walk on a square lattice, showing one hairpin. The crosses represent the obstacle lattice. The oval identifies a bead of the chain. While the hairpin diffuses past it, the bead is transported two steps along the tube toward configuration (d). (b) and (c) give intermediate configurations. For clearer representation we have opened the base of the hairpins. but only very slowly. It fully is developed only for surprisingly long chains $(N \gtrsim 100)$. It seems that for $N \longrightarrow \infty$, there is a well-defined limiting curve that merges with the expected power law only for Monte Carlo time $t_{\mathrm{MC}} \gtrsim 3 \cdot 10^{4}$. $\left(t_{\mathrm{MC}}=1\right.$ stands for one attempted move per bead of the chain.) For our longest chain $(N=$ $600)$ the $t^{1 / 4}$ power-law regime then extends over about two decades. For $N \lesssim 100$ the influence of the hairpins diffusing in from the ends of the chain becomes important, i.e., $T_{2}$ is exceeded before the power law properly is developed. The curves then bend upward according to Eq. (1). Effective exponents $\frac{1}{4}<x \leqq 0.4$ could easily be extracted for shorter chains, in full agreement with earlier observations.

To properly understand these results, we worked out the quantitative predictions of the original reptation model [1] in a fully discretized version. The defects are modeled as noninteracting particles, freely diffusing along a lattice chain. At the chain ends defects can be destroyed or created, which is modeled by coupling the ends to large reservoirs. If a defect passes a bead, that bead is displaced by two steps along the "tube", which is defined by the $t=0$ configuration of the chain (see Fig. 1). Let $n_{ \pm}(t, j)$ be the number of defects passing bead $j$ within time interval $t$ from the left $(+)$ or right $(-)$, respectively. Then $2 n(t, j)$ with

$$
n(t, j)=n_{+}(t, j)-n_{-}(t, j)
$$

gives the displacement of that bead along the "tube". If $t$ is short compared to the tube renewal or reptation time $T_{3}=\mathcal{O}\left(N^{3}\right)$, this effective diffusion for a central bead takes place in the fixed initial tube. Since this tube is a realization of a random walk on a cubic lattice with lattice constant $a=1$, i.e., with lattice vectors $\mathbf{s}_{i}$ with Cartesian components $s_{i}^{\mu} \in\{0, \pm 1\}$ and $\left\langle\mathbf{s}_{i} \cdot \mathbf{s}_{i^{\prime}}\right\rangle_{t}=$ $\delta_{i i^{\prime}}$, the average over all tube configurations yields for $t \ll T_{3}$ (a lower index $c$ denotes the average over tube

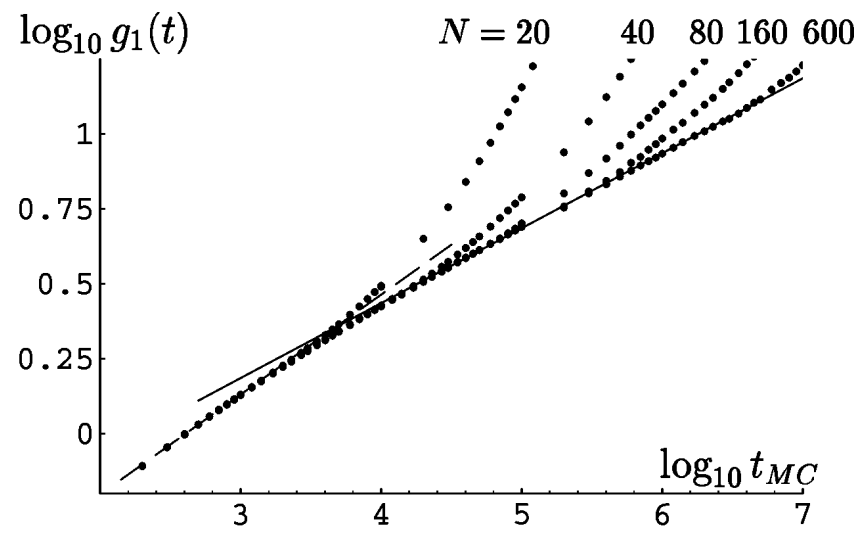

FIG. 2. Doubly logarithmic plot of Monte Carlo data for various chain lengths $N$. The straight lines give slope $1 / 4$ or $1 / 3$, respectively. 
configurations and $d$ over thermal defect dynamics)

$$
\begin{aligned}
g_{1}(t) & =\left\langle\left[\mathbf{r}_{N / 2}(t)-\mathbf{r}_{N / 2}(0)\right]^{2}\right\rangle_{c, d} \\
& =\left\langle\left(\sum_{i=1}^{2|n|} \mathbf{s}_{i}\right)^{2}\right\rangle_{c, d}=\left\langle\left|2 n\left(t, \frac{N}{2}\right)\right|\right\rangle_{d},
\end{aligned}
$$

a result that should hold up to a microstructure dependent additive constant $c_{0}$ much less than one. Such corrections, for instance, arise from configurations where the bead at times 0 or $t$ sits on the tip of a hairpin, such configurations being not included faithfully in our defect model. Note that we use the notion of the "tube" in a somewhat loose way, not distinguishing it properly from the chain configuration. In the MC model the tube can be defined as the chain configuration with all hairpins cut off, resulting in a nonreversal random walk. The simple random-walklike spatial embedding $\mathbf{r}$ of the internal chain coordinates implied by Eq. (3) is justified by the observation that both defects and bead move along the chain, not along the tube.
Our model is similar in spirit to the repton model proposed by Rubinstein [10], but somewhat closer to the original de Gennes model. It allows one to analytically calculate dynamical properties like the time dependent distribution function $\mathcal{P}(t ; n, j)$ of $n(t, j)$, while taking the proper defect dynamics at the chain ends into account. Leaving details for a forthcoming paper, we only present some results for the central bead $j=N / 2$. The second moment of the distribution of $n(t, N / 2)$ is found as

$$
\begin{aligned}
\left\langle n^{2}\left(t, \frac{N}{2}\right)\right\rangle_{d}=\rho_{0}[ & \frac{2 t}{N}+\frac{N}{6}+\frac{1}{3 N}+\frac{1}{N} \sum_{k=1}^{N-1}(-1)^{k} \\
& \left.\times\left(1-\frac{\cos ^{2} \frac{\pi k}{2}}{\sin ^{2} \frac{\pi k}{2 N}}\right) \mathrm{e}^{-4 t \sin ^{2} \frac{\pi k}{N}}\right],
\end{aligned}
$$

where $\rho_{0}$ is the density of defects. In Eq. (4) we recognize the sum over the Rouse modes of the chain, typical for such problems. $g_{1}(t)$ [Eq. (3)] is found as a function of $\left\langle n^{2}\right\rangle$ as

$$
g_{1}(t)=\langle|2 n|\rangle=\frac{\left(2\left\langle n^{2}\right\rangle\right)^{1 / 2}}{\pi}\left\{2 \sqrt{\pi}+\Gamma\left(-\frac{1}{2}, 2\left\langle n^{2}\right\rangle\right)-\int_{0}^{2\left\langle n^{2}\right\rangle} d x x^{-3 / 2} e^{-x}\left[\left(1-\frac{x}{2\left\langle n^{2}\right\rangle}\right)^{-1 / 2}-1\right]\right\},
$$

where $\Gamma(x, y)$ denotes the incomplete Gamma function. In Eq. (5) both the term $\Gamma(\cdots)$ and the integral are due to the discrete structure of the chain. Evaluating these results in the time regime $T_{0} \ll t \ll T_{2}$, which mathematically is defined by the limit $N \longrightarrow \infty$ and $1 \ll$ $t<\infty$ fixed, we find the intermediate asymptotics

$$
g_{1, \infty}(t)=4 \pi^{-3 / 4} \rho_{0}^{1 / 2} t^{1 / 4} \text {. }
$$

In the time regime $T_{2} \ll t \ll T_{3}$, defined as limit $N \longrightarrow$ $\infty, 1 \ll t / N^{2}<\infty$ fixed, we recover the $t^{1 / 2}$ behavior: $g_{1}(t)=4\left(\rho_{0} / \pi\right)^{1 / 2}(t / N)^{1 / 2}$. These are the well known asymptotic reptation predictions (1).

In comparing our quantitative results to the simulation data, we have to specify the density of defects $\rho_{0}$. Due to the occurrence of larger side loops (double hairpins, etc.), the mapping of hairpins onto defects is not one-toone, so that $\rho_{0}$ is an effective model parameter. From the equilibrium statistics of a random walk, it is found to be fixed within very close bounds: $\frac{1}{9} \lessgtr \rho_{0} \lesssim \frac{1}{4}$. The shape of our theoretical curves is not sensitive to the precise value chosen, which mainly can be absorbed into the time scale. We here take $\rho_{0}=1 / 4$. We also need to fix the additive constant $c_{0}$ introduced above. From analyzing the data for microscopic times $\left(t_{M C} \lesssim 30\right)$, we choose to subtract $c_{0}=0.1$ from the Monte Carlo data for $g_{1}(t)$. The only really free parameter is the scale $\tau=t / t_{\mathrm{MC}}$, relating the time variable $t$ of the analytical model to the Monte Carlo time $t_{\mathrm{MC}}$. By adjusting this single parameter to $\tau=0.01$, we find the results of Fig. 3. Note that since we divided out the intermediate asymptotics (6), this plot is much more sensitive to small deviations than the usual doubly logarithmic representation. In view of this we believe the agreement found for $N \geq 40$ to be truly remarkable.

We now can analyze in detail the origin of the long transient, which in Fig. 3 extends up to $t \approx 10^{3}$. According to Eq. (5), $g_{1}(t)$ depends on $t$ only via $\left\langle n^{2}(t, N / 2)\right\rangle$. Now it is found that $\left\langle n^{2}(t, N / 2)\right\rangle$ approaches its intermediate asymptotics $\left\langle n^{2}(t, N / 2)\right\rangle^{1 / 2}=\left(2 \rho_{0}\right)^{1 / 2}(t / \pi)^{1 / 4}$ for times as small as $t \geq 3$. The slow transient thus is due to the deviations of $\langle|n|\rangle$ from $\left(2\left\langle n^{2}\right\rangle / \pi\right)^{1 / 2}$ for small $\left\langle n^{2}\right\rangle$ as exhibited in Eq. (5). These deviations reflect the discrete structure of the chain. A simple toy model explains this effect: The fluctuating quantity $n(t, j)$ takes only integer values and its distribution rapidly looks like a discretized Gaussian. The width of this Gaussian, however, increases

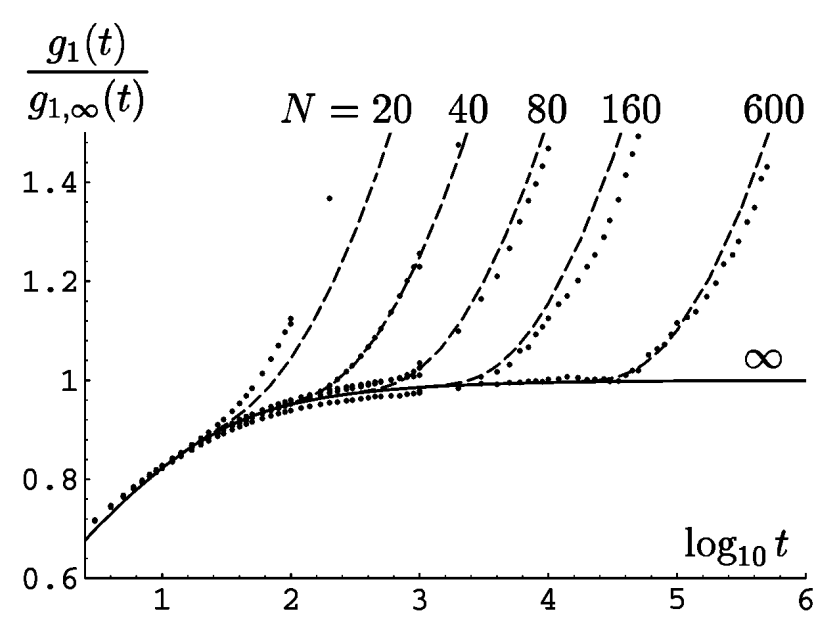

FIG. 3. $g_{1}(t) / g_{1, \infty}(t)$ plotted against $\log _{10} t, t=0.01 \cdot t_{\mathrm{MC}}$. Full and broken lines: theory. Points: MC data. 
but slowly: $\left\langle n^{2}(t, j)\right\rangle^{1 / 2} \sim t^{1 / 4}$. Even for $t \approx 10^{3}$ it shows nonzero weight essentially only for $|n| \lesssim 5$. Thus the discrete nature of the fluctuating variable $n(t, j)$ stays important for a very large initial time interval. Evaluating $\langle|n(t, j)|\rangle$ with such a discretized Gaussian, we recover the slow transient.

These considerations immediately suggest to eliminate the transient behavior by measuring $\left\langle n^{2}\right\rangle$ instead of $\langle|n|\rangle$. Now for the present model it is easily checked that the cubic invariant $\hat{g}_{1}^{2}(t)$ reduces to $\left\langle n^{2}\right\rangle$ :

$$
\begin{aligned}
\hat{g}_{1}(t) & =\left\langle\sum_{\mu=1}^{3}\left[r_{N / 2}^{\mu}(t)-r_{N / 2}^{\mu}(0)\right]^{4}\right\rangle_{c, d}^{1 / 2} \\
& =\left\langle\sum_{\mu=1}^{3}\left(\sum_{i=1}^{2|n|} s_{i}^{\mu}\right)^{4}\right\rangle_{c, d}^{1 / 2}=\left\langle 4 n^{2}\left(t, \frac{N}{2}\right)\right\rangle_{d}^{1 / 2} .
\end{aligned}
$$

For random-walk-type chains on other lattices or in the continuum the spatial embedding $\mathbf{r}$ of the basic dynamic quantity $\left\langle n^{2}\right\rangle$ differs, but $\left\langle n^{2}\right\rangle$ always can be expressed by an appropriate combination of the fourth and the second moment of $\left[\mathbf{r}_{N / 2}(t)-\mathbf{r}_{N / 2}(0)\right]$. Figure 4 shows a doubly logarithmic plot of our data for $\hat{g}_{1}$ as compared to $g_{1}$ for chain lengths $N=40$ and 600 . Clearly our expectation is born out. For $\hat{g}_{1}(t)$ only some slight initial deviation from the $t^{1 / 4}$ power law can be detected that could lead to a tiny underestimation of the exponent $(\approx 0.235$ in place of 0.25). Even for $N=40$ we find a decent $t^{1 / 4}$ regime, which for $N=600$ extends over almost four

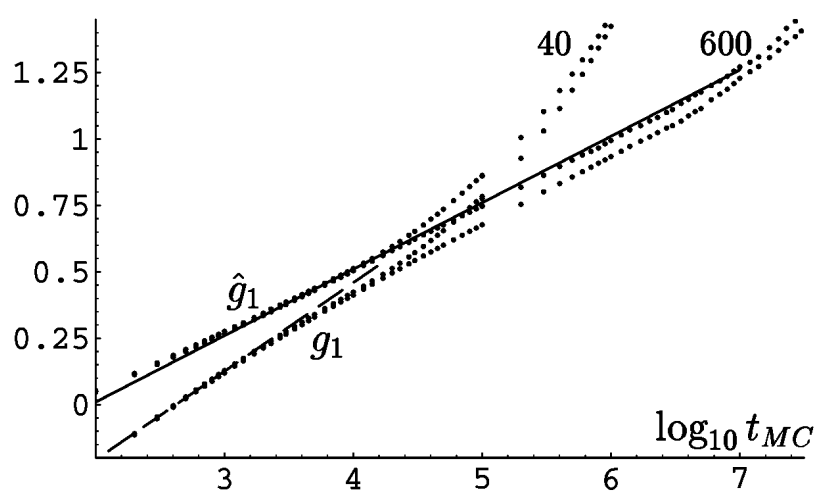

FIG. 4. Doubly logarithmic plot of MC-data (like in Fig. 2) for $g_{1}(t)$ and $\hat{g}_{1}(t)$ and for $N=40,600$. Full line: $t^{1 / 4}$ power law. Broken line: $t^{1 / 3}$ curve for comparison. decades. We thus conclude that our fourth moment shows asymptotic reptational behavior much more clearly than the commonly used second moment.

In summary, combining a simple exactly solvable model with computer experiments, we not only recovered the asymptotic reptation prediction of a $t^{1 / 4}$ regime, but we also quantitatively explained the shape of the crossover function with its long initial transient. Since any change of the microstructure of the model like an enlargement of the tube radius will only increase the initial effects, we believe our results to be typical also for other models. We have demonstrated that an appropriate fourth moment measuring $\left\langle n^{2}\right\rangle$ instead of $\langle|n|\rangle$ suppresses the initial transient and thus lends itself to a much simpler analysis. These results are hoped to provide a standard for the study of deviations from reptation due to disorder or due to a relaxation of the tube. In all cases we strongly recommend including the fourth moment of the displacement of the central bead into the analysis.

We thank J.M.J. van Leeuwen and D. Aalberts for helpful discussions. U.E. was supported by the Dutch Science Foundation NWO. Support by the SFB 237 of the German Science Foundation DFG is also gratefully acknowledged. The simulations have been performed at the Forschungszentrum Jülich.

[1] P. G. de Gennes, J. Chem. Phys. 55, 572 (1971).

[2] T. P. Lodge, N. A. Rotstein, and S. Prager, in Advances in Chemical Physics LXXIX, edited by I. Prigogine and S. A. Rice (Wiley, New York, 1990).

[3] A. Kolinski, J. Skolnick, and R. Yaris, J. Chem. Phys. 86, 7164 (1987).

[4] K. Kremer and G. S. Grest, J. Chem. Phys. 92, 5057 (1990).

[5] G. W. Slater and S. Y. Wu, Phys. Rev. Lett. 75, 164 (1995).

[6] U. Ebert, A. Baumgärtner, and L. Schäfer, Phys. Rev. E 53, 950 (1996).

[7] G. M. Foo, R. M. Pandey, and D. Stauffer, Phys. Rev. E 53, 3717 (1996).

[8] K.E. Evans and S.F. Edwards, J. Chem. Soc., Faraday Trans. 2, 1891 (1981).

[9] J. M. Deutsch and T. L. Madden, J. Chem. Phys. 91, 3252 (1989).

[10] M. Rubinstein, Phys. Rev. Lett. 59, 1946 (1987). 